\title{
A importância do STEAM frente aos desafios da formação do ensino superior e da pesquisa multidimensional em Design
}

\section{The importance of STEAM facing the new challenges of higher education and multidimensional research in Design}

\author{
Wilson Kindlein Junior, Departamento de Engenharia de Materiais (UFRGS) \\ wilsonkindleinjunior@gmail.com
}

Felix Bressan, Departamento de Artes Visuais (UFRGS)

felixbressan@gmail.com

Felipe Luis Palombini, Doutor em Design (PGDesign/UFRGS)

felipe.l.palombini@gmail.com

Resumo

O STEAM é uma das principais práticas da atualidade no que diz respeito a uma abordagem transdisciplinar envolvendo diferentes campos do conhecimento. Com as novas perspectivas de avaliação da graduação e da avaliação multidimensional da pós-graduação em Design no Brasil, surgem desafios na formação do estudante no que tange sua integração com diferentes locais de fala e sua participação em ambientes de pesquisa. Neste trabalho, refletimos sobre a importância da prática do STEAM em pesquisas em Design, através de casos de aplicação de tecnologias 3D de investigação, representação e fabricação. Apresentamos um exemplo prático da ligação entre as áreas por meio do desenvolvimento de um objeto de uso cotidiano com um software generativo e impressão 3D, referenciado no binômio arte-tecnologia, advindos do diagrama matemático de Voronoi que se faz presente em muitos elementos naturais. Por fim, é mostrado o papel do STEAM na integração de áreas e frente aos novos critérios da avaliação multidimensional, bem como a importância dos ambientes de pesquisas capazes de abarcar essas tecnologias, fornecendo, assim, ao aluno um estímulo e o ambiente necessário à sua formação de excelência.

Palavras-chave: STEAM, Transdisciplinaridade, Avaliação multidimensional

\begin{abstract}
STEAM is one of today's main practices concerning a transdisciplinary approach involving different fields of knowledge. With the new perspectives for evaluating undergraduate courses and a multidimensional evaluation of graduate programs in Design in Brazil, challenges arise in the students' training regarding their integration with different standpoint speech and their participation in research environments. In this work, we reflect on the importance of the practice of STEAM in Design research, through cases of $3 D$ technologies in the investigation, representation, and manufacturing. We present a practical example of link among areas through the development of a daily use object with generative software and 3D printing, referenced in the art-technology binomial, arising from Voronoi mathematical diagram. Finally, the STEAM role is showed in the integration of areas and facing the new criteria of multidimensional evaluation, and the importance of research environments capable of embracing such technologies, thus providing students with a stimulus and the necessary environment for their excellence in training.
\end{abstract}

Keywords: STEAM, Transdisciplinarity, Multidimensional evaluation 


\section{Introdução}

No cenário atual do mundo em constantes mudanças, existem muitos desafios na associação entre formação e atuação dos profissionais de nível superior. Para que a atuação profissional integre a responsabilidade social é fundamental que a formação seja multidimensional, abarcando não apenas conceitos técnicos, mas também uma compreensão compartilhada baseada numa vida em comum, visando a uma sociedade mais justa, fraterna e igualitária. A formação multidimensional exige um olhar transdisciplinar. A transdisciplinaridade está vinculada com a adoção de práticas oriundas de diversas áreas do conhecimento e diferentes campos de fala. "A transdisciplinaridade não procura o domínio de várias disciplinas, mas a abertura de todas as disciplinas ao que as une e as ultrapassa" (FREITAS; MORIN; NICOLESCU, 1994). É comum a falsa identificação de similaridade com termos como multidisciplinaridade e interdisciplinaridade (QUIGLEY; HERRO; BAKER, 2019). Uma prática multidisciplinar é atribuída quando especialistas de diferentes disciplinas trabalham de maneira independente e paralela em aspectos distintos de um projeto, permanecendo em suas próprias áreas (MALLON; BUNTON, 2005). Uma estrutura interdisciplinar é semelhante, contudo, tem como mote principal unificar duas ou mais disciplinas para criar uma nova disciplina híbrida, ou uma 'interdisciplina' (SCHUMMER, 2004). Já a educação transdisciplinar ocorre quando o conhecimento é tratado holisticamente, mesmo ainda quando apoiada em uma disciplina-base, reconhecendo diferentes pontos de vistas, suposições e descobertas (QUIGLEY; HERRO; BAKER, 2019). Além de práticas de ensino, o desenvolvimento de pesquisas transdisciplinares também possui como fator fundamental a interação de profissionais distintos em ambientes de trabalho em comum (KINDLEIN JÚNIOR, 2014). Ter uma visão transdisciplinar significa ultrapassar o campo das ciências e intregar no projeto o imaginário vindo das artes visuais, da literatura, da poesia, da música e de quaisquer outros saberes e competências que possam agregar conceitos que contemplem um olhar holístico, com todas as suas especificidades e sensibilidades.

Uma das principais práticas que vai ao encontro da transdisciplinaridade é a atualmente conhecida pelo acrônimo STEAM, do inglês Science, Technology, Engineering, Arts e Mathematics. Prática originalmente advinda do SMET (Science, Mathematics, Engineering, e Technology) pelo National Science Foundation (NSF) nos anos 1990, a sigla foi reordenada para STEM (Science, Technology, Engineering e Mathematics), pela bióloga americana Judith Ramaley em 2001 (HALLINEN, 2019). Já adição do termo 'Arts' na sigla, nos anos seguintes, tem sido uma prática cada vez mais defendida por educadores que veem como um estímulo à criatividade e à inovação (GUYOTTE et al., 2014). Além da adição das chamadas 'Artes Liberais' à prática de ensino, alguns autores (GE; IFENTHALER; SPECTOR, 2015; LIAO, 2016; PERIGNAT; KATZ-BUONINCONTRO, 2019) ainda defendem que o 'A' da sigla também seja representativo das áreas relacionadas a humanidades. No presente artigo defendemos, inclusive, que o design possa ser o elo entre estes vários campos do conhecimento (figura 1). Para tal, é fundamental agregar nesse arcabouço de saberes e competências os novos paradigmas de processos de investigação científica, fabricação e materiais. Dentre eles, o uso das tecnologias 3D que auxiliam as práticas de STEAM em currículos de ensino superior em design, de maneira a permitir processos de criação e desenvolvimento mais aprimorados e até mesmo inovadores (CHIEN; CHU, 2018; PALOMBINI et al., 2018a). Somado a essas observações, com o advento 
das novas ferramentas computacionais de caracterização, modelagem e fabricação digital, tornase possível a agilidade na experimentação de ideias e, até mesmo, podem surgir alternativas inesperadas devido à retroalimentação durante o processo criativo.

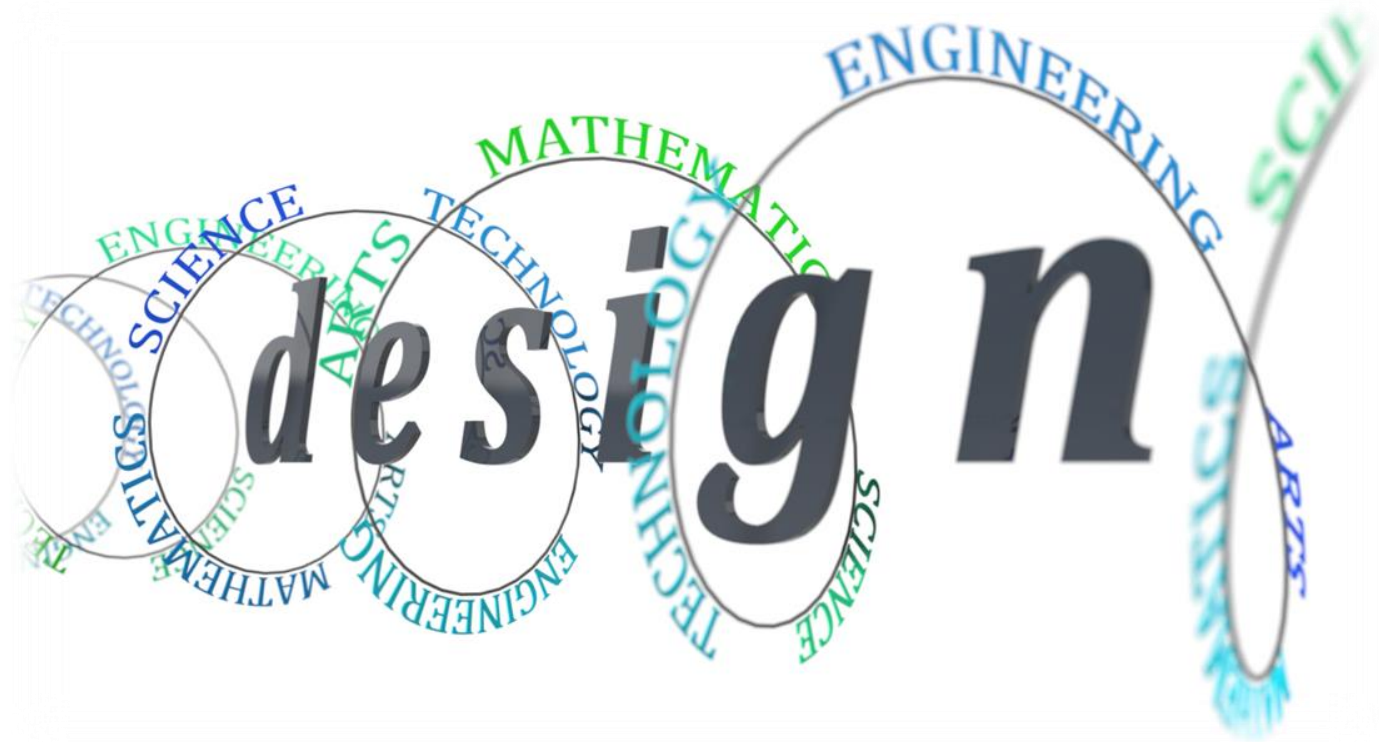

Figura 1: Proposta de STEAM como uma prática guiada pelo design. Fonte: Autores (2021).

Muitos dos estudos relacionados à prática do STEAM estão focados no ensino básico ou médio, como defendido pela educadora Georgette Yakman (STEAM EDUCATION, 2016), de modo a serem um meio de estimular processos criativos entre jovens (HARRIS; DE BRUIN, 2018; SHATUNOVA et al., 2019). Contudo, é importante destacar também a prática do STEAM para estudantes de esino superior como um modo dos professores trabalharem não apenas o pensamento criativo, mas aprimorar habilidades de resolução de problemas, consideradas uma "nova demanda do século XXI" (MARMON, 2019). Lewis (2015), por exemplo, destaca três paradigmas principais da adoção de práticas do STEAM no ensino superior: investigação, interatividade e inovação. O primeiro diz respeito à adição de meios computacionais na obtenção de conhecimento. $\mathrm{O}$ segundo trata da integração entre das Artes visuais com o processo cognitivo. Já o último está relacionado a elaboração de um ambiente transdisciplinar, com aprendizagem realizada por meio do pensamento iterativo, no sentido de um processo retroalimentado, com idas e vindas de processos em busca de inovação. Neste sentido, o presente estudo tem como intensão reforçar a importância do uso do STEAM em práticas de ensino e pesquisa no que tange aos desafios emergentes da formação do ensino superior em Design. São apresentados exemplos de como as práticas transdisciplinares e a utilização de tecnologias 3D contribuem para a obtenção de resultados inovadores em pesquisas. Por fim, o STEAM é cotejado frente aos desafios da Avaliação Multidimensional da CAPES, bem como o potencial de inovação do STEAM é abordado no contexto de desenvolvimento do ensino superior de Design no país.

\section{O Design e a transdisciplinaridade em pesquisa}

A integração de áreas distintas em uma perspectiva transdisciplinar tem um papel fundamental no desenvolvimento de pesquisas inovadoras em Design. Ao realizar a integração de conhecimentos torna-se possível buscar diferentes pontos de vista sobre um determinado tema, o 
que ajuda a interpretar a problemática de uma maneira holística. Recentemente, com o advento e com um maior acesso às tecnologias 3D, pesquisas em diversas áreas têm se beneficiado do seu potencial de exploração. Uma das principais técnicas de investigação tridimensional de alta resolução da atualidade é a microtomografia computadorizada de Raios X, ou $\mu$ CT (PALOMBINI et al., 2020b). A $\mu$ CT funciona de maneira minimamente invasiva por meio da digitalização de amostras com base em projeções sequenciais, que se aproveitam das propriedades da radiação no comprimento de onda de Raios X ser atenuada por regiões de diferentes densidades. As projeções 2D são unificadas em uma reconstrução 3D através dos princípios matemáticos da transformada de Radon, em homenagem ao matemático austríaco Johann Radon, que demonstrou em 1917 que um objeto pode ser replicado a partir de um conjunto infinito de suas projeções (HARRER; KASTNER, 2011). Modelos obtidos por $\mu \mathrm{CT}$ podem ser utilizados em diversas aplicações, como para estudos nas ciências biológicas e da saúde, no desenvolvimento de materiais, em análises estruturais, na indústria eletrônica, entre outros (STOCK, 2009). Além disso, devido à sua característica tridimensional e volumétrica (não superficial), os modelos podem tanto ser rematerializados em diferentes escalas por meio de tecnologias como a manufatura aditiva (PALOMBINI et al., 2017, 2018b), quanto serem empregados em análises físico-mecânicas por meio do Método de Elementos Finitos (FEM). Inicialmente estudado como um método para resolução de equações diferenciais, combinando o trabalho de inúmeros pensadores, como o físico e matemático suíço Leonhard Euler e do polímata e filósofo alemão Gottfried Leibniz, o FEM trata basicamente da divisão de sistemas complexos - considerados de natureza quase 'infinita' devido às inúmeras variáveis - em um conjunto finito de subsistemas (PALOMBINI et al., 2020a; STEIN, 2014). Por sua vez, tais subsistemas podem ser resolvidos mais facilmente, sendo posteriormente unificados para uma resolução total aproximada do sistema original. Esse método, que utiliza um número finito de elementos - conforme o nome - permitiu o desenvolvimento de projetos complexos e o crescimento tecnológico de diversas indústrias, como a aeroespacial, automobilística, de construção civil, entre outras (ZIENKIEWICZ; TAYLOR; ZHU, 2013).

Além do desenvolvimento de novos processos, a integração de áreas por meio de pesquisas transdisciplinares tem sido importante para a quebra de paradigmas em ciências clássicas e centenárias, como por exemplo a Botânica. A exemplo, um estudo de caso da tese de Doutorado (PGDesign/UFRGS) de Felipe Luis Palombini (2020), intitulada "Diretrizes para pesquisas em materiais vegetais com análises por elementos finitos baseadas em microtomografia de raios $\mathrm{X} \mathrm{e}$ implicações para projetos de biônica em design e engenharia", aborda a utilização das técnicas de $\mu \mathrm{CT}$ e FEM para estudo do papel biológico da inflorescência-tanque de determinadas espécies de Bromeliaceae. Foi conduzido um estudo experimental preliminar, com um determinado grupo amostral com indivíduos sem a presença de água no tanque, em comparação com um grupo de controle (com água no tanque). Verificou-se a presença de danos fisiológicos nos indivíduos do grupo experimental em decorrência da ausência de água no tanque, mesmo estando com a presença de água na terra e nas folhas. Por tanto, foi levantada a hipótese (NOGUEIRA et al., 2019) de que a inflorescência-tanque trabalha não como uma fonte de água para a planta, mas como um regulador térmico para as gemas florais. Para investigar o papel da água na regulação térmica dessa espécie, uma amostra foi digitalizada em alta resolução por $\mu \mathrm{CT}$, de modo a gerar um modelo 3D da região do tanque. Este modelo foi transformado em uma malha de elementos finitos e aplicado em um ensaio virtual de transferência de calor, de modo a simular os efeitos 
térmicos da utilização, ou não, de água no interior da inflorescência-tanque. Com a análise térmica da inflorescência, foi verificado que a água atua como um elemento de massa térmica, regulando a temperatura dos tecidos da planta de modo muito superior do que se o tanque estivesse vazio (PALOMBINI, 2020). Partindo da confirmação da função biológica da inflorescência-tanque de determinados gêneros de Bromeliaceae, esta característica foi aplicada pelo autor no desenvolvimento de uma garrafa térmica, aplicando a metodologia de biônica (KINDLEIN JÚNIOR; GUANABARA, 2005). A proposta consistiu na aplicação de um elemento de núcleo no interior de uma tampa externa da garrafa, sendo definido como um material com propriedades tanto de baixa condutividade térmica quanto de alta capacidade térmica. $\mathrm{O}$ modelo foi analisado novamente utilizando o modo de transferência de calor via elementos finitos, sendo confirmado que a presença de um material de núcleo aumentou o desempenho de isolamento térmico da garrafa quando em comparação com uma garrafa referencial, sem a presença do componente.

Outro exemplo importante de integração de áreas, no que tange o conceito de STEAM é a tese de Doutorado (PGDesign/UFRGS) de Felix Bressan (2018), intitulada "Design e tecnologia: estratégias generativas expressivas em superfície e volumetria". Nesta tese, uma das esculturas apresentadas trata da aplicação dos padrões de preenchimento Voronoi. A figura 2 apresenta um exemplo de inspiração para a geração de um padrão Voronoi baseado em uma micrografia do arranjo celular do felema de Quercus suber (cortiça).
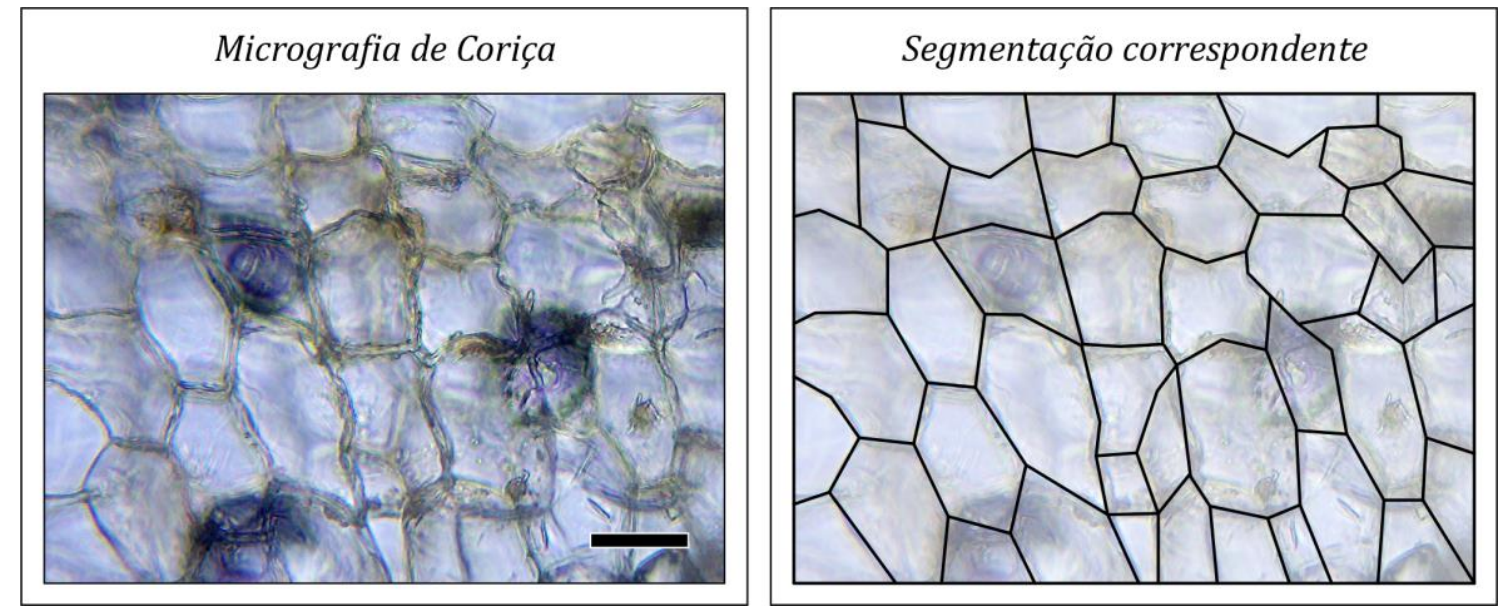

Figura 2: Exemplo de micrografia de arranjo celular de cortiça $(Q$. suber $)$ e processo de segmentação correspondente. Barra de escala é $20 \mu \mathrm{m}$. Fonte: Autores (2021).

O diagrama de Voronoi pode ser resumido de maneira geral como um algoritmo matemático alusivo a um padrão encontrado inúmeras vezes na natureza para dividir superfícies em espaços geométricos segmentados (OGNIEWICZ; ILG, 1992). É gerada uma serie de polígonos de número de lados variáveis, geralmente de 4 a 8 lados. Esses polígonos têm um ponto gerador que pode ser chamado de semente. As distâncias entre as bordas dos polígonos adjacentes são equidistantes de seus pontos geradores. Podemos mudar a distribuição desses pontos conseguindo assim configurações variadas, possibilitando alterações estruturais e estéticas. A figura 3 mostra dois exemplos de diagrama de Voronoi, gerados no software Rhinoceros ${ }^{\circledR} 3 \mathrm{D}$ (McNeel ${ }^{\circledR}$, Seattle, EUA). As imagens apresentadas possuem diferentes densidades de pontos. $\mathrm{O}$ primeiro diagrama foi gerado com 26 pontos (sementes), distribuídos aleatoriamente, e o segundo foi gerado duplicando-se o número de pontos, ou seja, com 52 sementes, com distribuição também aleatória. 

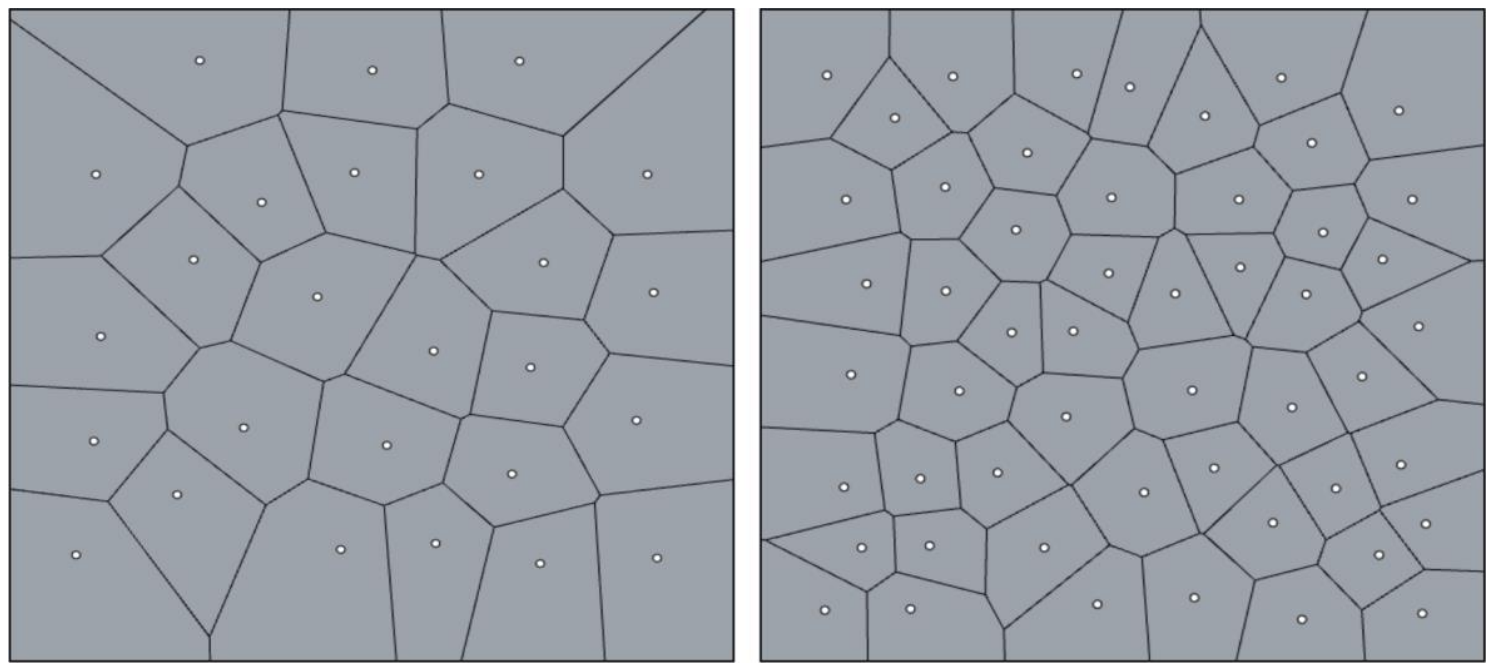

Figura 3: Exemplos de diagrama Voronoi com diferentes densidades de sementes. Fonte: Autores (2021).

Partindo-se, então, da natureza como referencial, segue inicialmente um exemplo do uso do diagrama de Voronoi no campo das artes. Na escultura da figura 4, ao mesmo tempo em que é criada uma trama com orifícios e ligações, estruturalmente, todo peso da peça é suportado pelo conjunto de segmentos que a compõe. Os módulos se relacionam de forma harmônica, fixados entre si; nessa obra, com o auxílio de parafusos. Esse projeto tem um discurso que apresenta um espaço vazio que seria ocupado por um suposto corpo que não existe fisicamente, mas sim no imaginário do expectador. Essa obra faz parte da série "O Corpo Ausente", trabalho artístico desenvolvido por Felix Bressan.
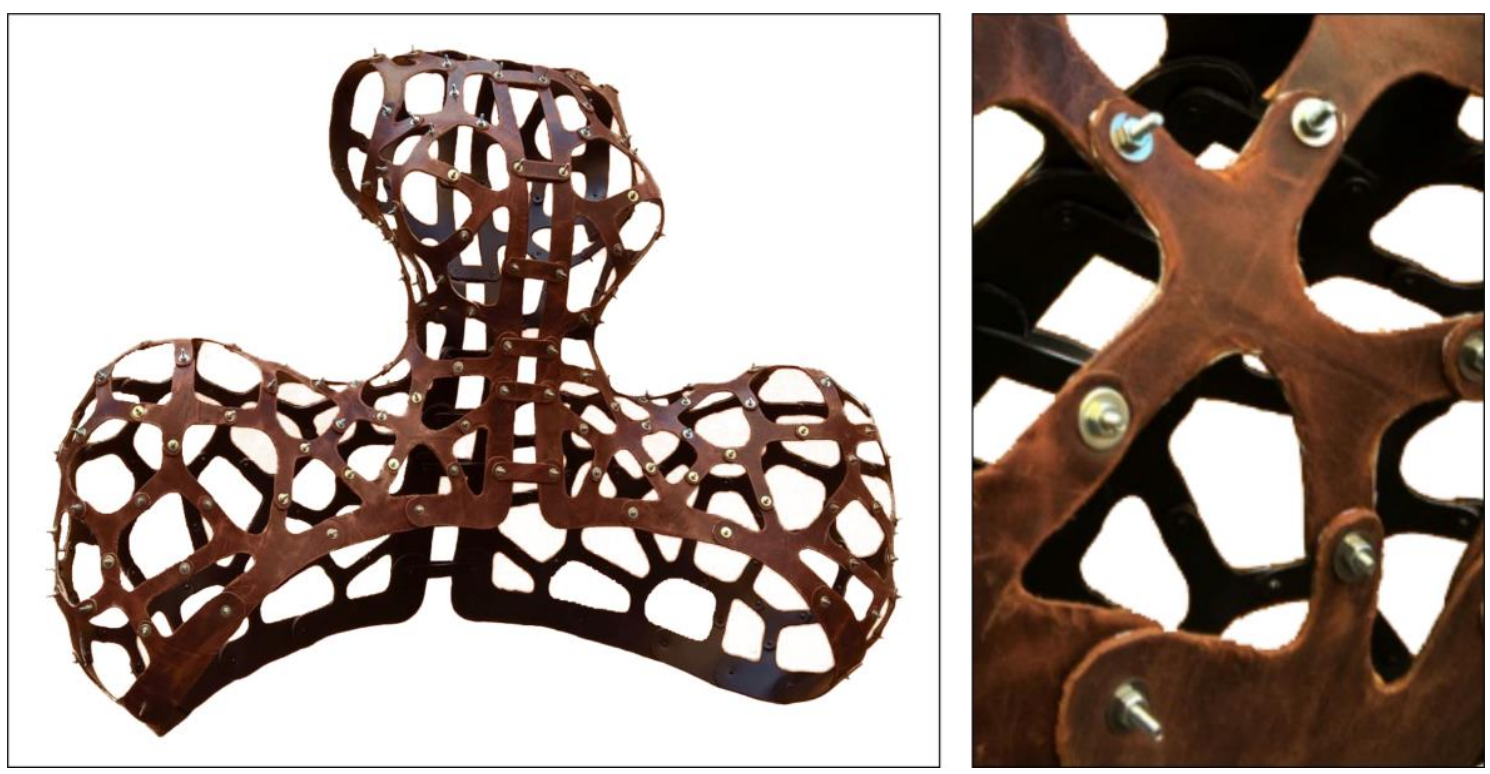

Figura 4: Escultura e detalhe da série "O Corpo Ausente", de Felix Bressan, como exemplo de aplicação do diagrama de Voronoi. Fonte: Autores (2021).

É interessante trazer para o contexto deste estudo da integração entre ciência, tecnologia, engenharia, artes e matemática (STEAM) que o conhecimento (savoir-faire) do modus operandi de mestres de ofício (alfaiates, sapateiros, marceneiros, caldeireiros etc.) traz referências fundamentais de base para a criação de novos produtos levando em conta o binômio "Memória \& Novidade". Há uma expressão que diz: "para mudar é necessário, antes, permanecer". Neste 
sentido, conhecer as técnicas, os materiais e os processos de mestres e artífices de ofício, bem como de empresas tradicionais têm forte influência na abertura de novos caminhos para unir, por exemplo, arte \& tecnologia, arte \& ciência, arte \& matemática, etc. É muito importante conhecer o processo histórico pelo qual esses saberes e competências se desenvolveram, ou como acabaram e/ou como chegaram até os dias atuais. Nesta seara, vale salientar a dissertação do designer Manlio Maria Gobbi (2011), intitulada "Design e tecnologia: resgatando ícones de inovação em móveis para escritório via arqueologia industrial". A área de arqueologia industrial é uma das bases para que possamos unir tradição \& inovação. Nesta mesma linha de pensamento, aportar um novo olhar para materiais antigos (por exemplo: o arenito) fazendo uso dos novos processos de fabricação (por exemplo: a gravação e o corte a laser) nos leva a caminhos interessantes no que diz respeito a união do antigo ao novo. Esse é o caso da dissertação da designer Tamara Barbian (2015) defendida no PGDesign/UFRGS, intitulada "Design e tecnologia: gravação de desenhos a laser no arenito". Essa abordagem integradora dos saberes e competências antigos e atuais é outra fonte que podemos beber quando estamos procurando fazer frente aos novos desafios da formação multidimensional do ensino superior em Design.

Alguns trabalhos recentes também têm apresentado abordagens transdisciplinares na geração de alternativas de projetos inovadores. A exemplo, no artigo de Fagundes et al. (2020) foi estudada a aplicação da gramática de forma de frutos de casca dura como o da palmeira Jupati na geração de alternativas para projetos de painéis para fachadas de edifícios. Os autores utilizaram tecnologias de investigação de variadas complexidades desde fotografia macro à $\mu \mathrm{CT}$, aplicando as informações levantadas na geração de sistemas e subsistemas por meio da biônica. Neste estudo, com o auxílio do software Grasshopper ${ }^{\circledR}$, foi proposto um conjunto de formas com sistemas de abertura e fechamento para aplicação nas fachadas. Bugin et al. (2020) também utilizaram este software para geração de alternativas no projeto de entressola de calçados; neste estudo os autores utilizam modificações de escalas do diagrama Voronoi e o materializam nas entressolas através de impressão 3D.

Para exemplificar melhor e contextualizar a importância de estar aberto a novas possibilidades criativas advindas de desenvolvimentos inesperados que unem o "novo ao velho" um case histórico pode ser aqui apresentado, focalizando no caso específico da produção do produto "luvas" (vale informar que a cidade de Grenoble na França, no século XIX, tinha metade de sua população trabalhando nesta fileira produtiva). A cidade é, até hoje, conhecida como a capital mundial da produção de luvas de luxo. No livro intitulado "Grenoble, cité internationale, cité d'innovations - rêves et réalités" (BLOCH, 2011) é possível perceber a importância dos materiais e do inusitado na criação de novos produtos (Material Driven Design, ou Design Orientado pelo Material). No início do século XX, o senhor Valérien Perrin, proprietário de uma grande fábrica de luvas em Grenoble (França), iniciou pesquisas para usar a viscose em sua fábrica. Sua empreitada, com o uso deste material não obteve o sucesso que ele esperava, mas seu filho Paul resolveu utilizar a viscose para produzir roupas íntimas (lingeries). Essa iniciativa, corajosa e criativa, deu origem, em 1925, a criação da fábrica de lingeries Valisère ${ }^{\circledR}$ que ficou mundialmente conhecida tempos depois.

Esse olhar diferenciado sobre as propriedades dos materiais e processos de fabricação e suas possíveis novas aplicações (mesmo que disruptivas) tem forte potencial para gerar inovação. 
Neste caso específico, o material, que não foi bem-visto como alternativa para a confecção das luvas (onde o material aceito e consolidado era a pele de cabra), foi redirecionado para a produção de lingeries, ou seja: uma solução inesperada para o uso inicialmente previsto.

É possível mencionar outro exemplo de ação corajosa e criativa, partindo do produto

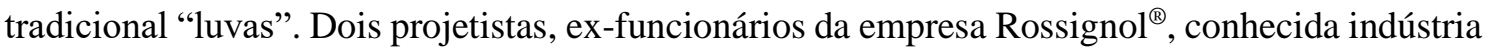
francesa de material esportivo de inverno e de montanha, imaginaram o produto "luva" como um novo suporte artístico. Em 2008 fundaram a FST HandWear ${ }^{\circledR}$ (https://www.fsthandwear.com) que utiliza como material a microfibra (um fio sintético, extremamente fino, geralmente de poliéster, poliamida ou acrílico) e como técnica de fabricação a sublimação para ornamentar suas coleções de luvas. Essa empresa tem forte aderência com a temática em estudo, pois vincula arte, design e tecnologia no desenvolvimento das novas coleções. Outra referência muito interessante na área de STEAM é o salão "Experimenta - Biennal Arts Sciences" (a edição mais recente ocorreu em 2020). Este evento agrega várias atividades diretamente vinculadas ao domínio do STEAM. Todas as obras apresentadas no salão têm foco no binômio artes e ciências sendo que a maioria é fruto de residências de artistas em conjunto às indústrias e a grupos de pesquisa científica e tecnológica.

Com o intuito de apresentar, na prática, o uso do STEAM aqui neste trabalho, três diferentes capas de telefones celulares (figura 5) foram projetadas a fim de demonstrar a potencialidade e incentivar o uso do STEAM no desenvolvimento de estratégias criativas que integrem diferentes campos de conhecimento (ciência, tecnologia, engenharia, artes e matemática), tendo como fio condutor o design. Para abrangermos vários aspectos inerentes à criação, aplicando o conceito STEAM, foi utilizado o editor de algoritmos gráficos Grasshopper ${ }^{\circledR}$ que foi implementado em 2007 por David Rutten, na empresa McNeel \& Associates (WALLISS; RAHMANN, 2016).

O Grasshopper ${ }^{\circledR}$ trabalha sobre a plataforma do Rhinoceros ${ }^{\circledR}$. Atualmente já está integrado como ferramenta nativa na plataforma, mas inicialmente utilizava o conceito de plug-in. Com ele, é possível executarmos as etapas e ferramentas que a plataforma disponibiliza e outras, externas, que foram implementadas por terceiros. Isso agrega e deixa muito versáteis processos de modelagem e criação em 3D. A ferramenta permite desenvolver projetos com o conceito de modelagem paramétrica, em que objetos são criados através de parâmetros pré-configurados, facilitando a realização de alterações no modelo. Todas as etapas do processo de criação ficam registradas de maneira amigável, como em uma fila de comandos. Esse método também permite utilizar o software como uma ferramenta de auxílio à criatividade, pois, muitas vezes resultados que não são esperados ou não pensados podem ser desenvolvidos durante o processo de execução do modelo, gerando, assim, uma série de opções não previstas inicialmente pelo usuário.

Por suas características peculiares, o Grasshopper ${ }^{\circledR}$ pode ser um dos elos que permitem a integração entre diferentes áreas do conhecimento. Poderíamos dizer, de uma outra forma, que alguns projetos que utilizam as instâncias que compõem o STEAM (Ciência, Tecnologia, Engenharia, Arte e Matemática) podem ser integrados através do Design com o auxílio da plataforma de desenvolvimento Rhinoceros ${ }^{\circledR}-$ Grasshopper $^{\circledR}$. Essa é uma boa e preocupante notícia para os criadores, pois ao mesmo tempo em que se apresentam como recursos que aumentam a possibilidade criativa e a velocidade de execução das tarefas, também faz-se 
necessário o aprendizado dessas ferramentas de maneira que as mesmas possam ser incorporadas na caixa de conhecimento dos designers, engenheiros, artistas, etc.
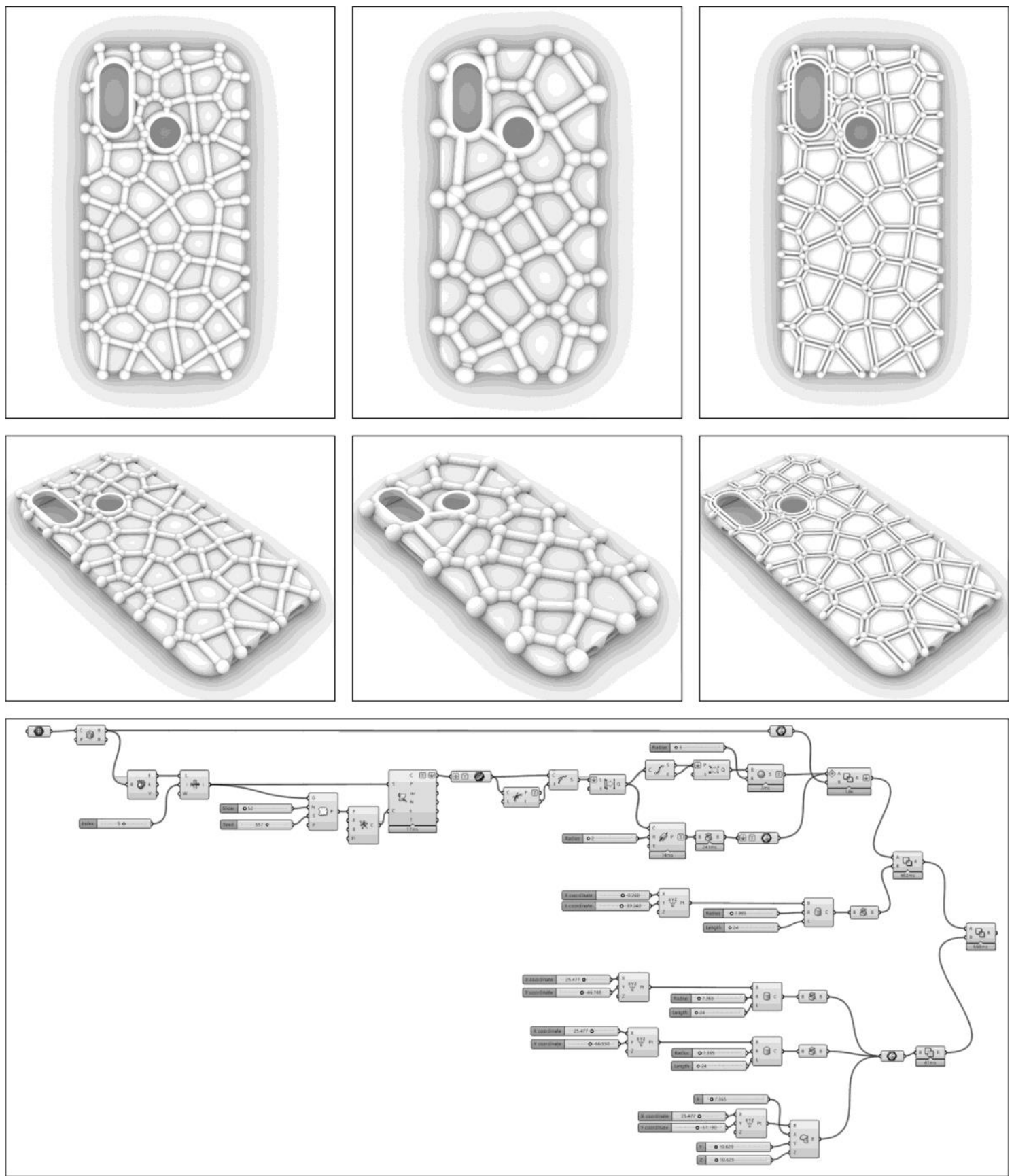

Figura 5: Exemplo de aplicação de conceitos do STEAM. Diferentes variações de capas de celular gerada com o software Grasshopper $^{\circledast}$, utilizando de preenchimento de Diagrama de Voronoi. Fonte: Autores (2021).

A manufatura aditiva é uma das grandes aliadas do designer na materialização de seus projetos, desde mock-ups a produtos finais. Com o intuito de concretizar fisicamente a ideia, foi utilizado o equipamento marca ANYCUBIC ${ }^{\circledR}$ 3D modelo Photon (Shenzhen Anycubic Technology Co. Ltd., Shenzhen, China), que utiliza a tecnologia LCD-based SLA (estereolitografia que usa uma fonte de luz ultravioleta proveniente de uma série de LEDs). A figura 6 apresenta o processo de fabricação 3D utilizado neste trabalho a fim de demonstrar a importância dessas tecnologias como ferramentas rápidas que auxiliam na materialização das ideias. Vale salientar que a intensão não 
é aqui selecionar o material mais indicado e tão pouco o processo de fabricação mais adequado para este fim específico, mas vale mostrar o equipamento que foi empregado e alguns detalhes dos protótipos estéticos obtidos. A fabricação destes mock-ups (65\% do tamanho real de uma capa de celular, devido às limitações do tamanho da área útil de impressão do equipamento utilizado) foi realizada com objetivo da verificação estético-formal, para se ter uma ideia visual dos parâmetros geométricos virtuais gerados na aplicação do diagrama de Voronoi. É mister, consequentemente, que este trabalho não teve a intensão de realizar o estudo das propriedades estruturais requeridas para a manufatura de um possível protótipo funcional. Cabe destacar ainda que um modelo em tamanho real poderia ser impresso com a divisão do objeto em partes e a elaboração de sistemas de encaixes, além de que análises adicionais de espessura de material e de desempenho mecânico da resina fotopolimerizada poderiam ser realizados com o objetivo de definir os melhores parâmetros para aplicação em um modelo funcional.
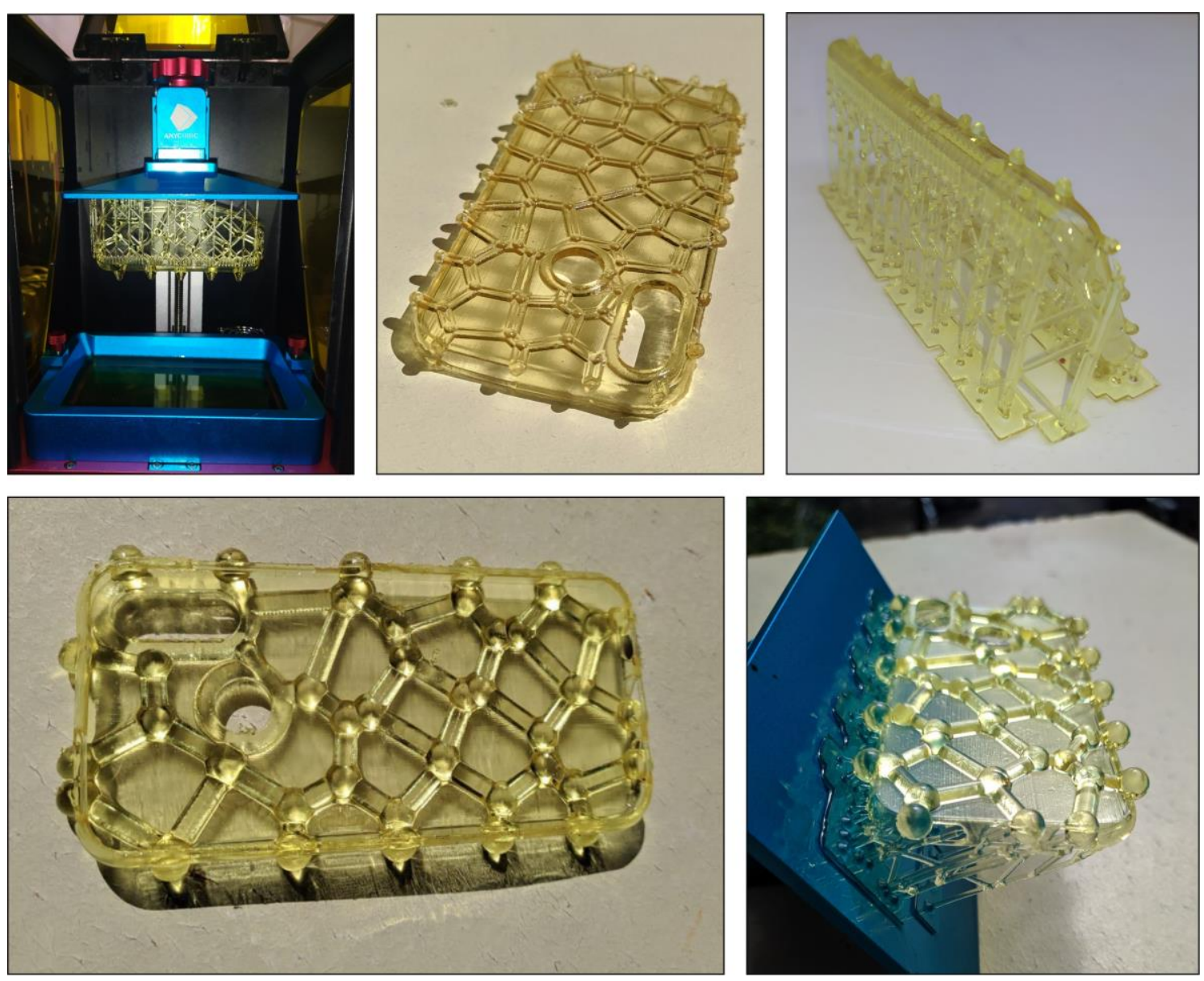

Figura 6: Processo de fabricação por impressão 3D de modelos desenvolvidos das capas de celular. Fonte: Autores (2021).

O funcionamento do equipamento consiste na divisão do modelo a ser impresso em camadas, sendo que cada uma é projetada em uma tela de LCD de alta resolução na parte inferior transparente de um compartimento contendo uma resina fotossensível, no seu interior. A luz projetada pela tela LCD polimeriza a resina e solidifica a peça, camada por camada. Deste modo, ainda que não sejam considerados protótipos funcionais, devido aos materiais, processos e fatores ergonômicos, a técnica permitiu a visualização física do modelo com uma grande precisão de detalhes, com espessura de camada definida em $50 \mu \mathrm{m}$ (20 camadas por milímetro). 


\section{STEAM no contexto da Pós-Graduação em Design}

A avaliação da pós-graduação com caráter "multidimensional" que está sendo discutida na CAPES para o quadriênio 2021/2024 reforça a necessidade de tratar de maneira interligada diferentes "campos de fala". As cinco dimensões (eixos) que serão foco de atenção na próxima avaliação da pós-graduação no Brasil são: 1. ensino e aprendizagem; 2. produção de conhecimento; 3. impacto e relevância para a sociedade; 4. internacionalização e 5. inovação e transferência de conhecimento. Neste sentido, enfoques de cunho multidimensionais que interligam diferentes campos de conhecimentos vêm ao encontro da necessidade de abordar de forma conjunta o ensino, a pesquisa e a extensão em Design. É mister, porém, que estas ações sejam realizadas com responsabilidade social e excelência acadêmica. Essa premissa robustece a importância de uma abordagem técnico/científica holística de enfrentamento da temática em questão. Sendo assim, fica evidente que há um forte potencial de aderência do STEAM frente aos imperativos do trabalho conjunto que se faz necessário na produção multi-atores que caracteriza o atual cenário do desenvolvimento de novos produtos e serviços. Somado a esse fato, com o intuito de avançar com suas políticas de incentivo aos programas de mestrado e doutorado stricto sensu de caráter industrial, a avaliação quadrienal do Plano Nacional da Pós-Graduação - PNPG e o CNPq através da chamada MAI-DAI-CNPq, pretendem elaborar, avaliar e revisar seus critérios (BRASIL, 2017; PNPG 2017). As ações encorajadas pelo conceito de STEAM dão asas às ideias, aportam ânimo para a tomada de riscos e permitem o aprendizado com os erros. $\mathrm{O}$ STEAM encadeia a transdisciplinaridade, a interdisciplinaridade, a multidisciplinaridade e mesmo as disciplinas-cruzadas (PERIGNAT; KATZ-BUONINCONTRO, 2019). Essa é uma razão a mais para reforçar a importância de abordar os trabalhos de graduação, mestrado e doutorado com um olhar multidimensional de forma a interligar diferentes corpos de conhecimento e agregar diversos campos de fala.

\section{Considerações Finais}

O presente artigo traz contribuições que reforçam a importância das práticas pedagógicas transdisciplinares frente aos desafios da formação do ensino superior e da pesquisa em Design. O trabalho enfatiza uma visão multidimensional na formação do designer, o que preconiza ambientes colaborativos de desenvolvimento de produtos e serviços, bem como tem aderência à ênfase da futura avaliação dos cursos de graduação e de pós-graduação do sistema educacional no Brasil.

Nossa conclusão é de que a abordagem STEAM permite ao designer associar diferentes campos de conhecimento na busca da integração de saberes e competências. O STEAM vai ao encontro das novas possibilidades advindas das atuais tecnologias de fabricação, modelagem e representação. O entendimento dos materiais e dos processos é fundamental para a formação do designer e necessitam ser bem integrados na sua formação, pois permitem materializar uma ideia à luz das ferramentas científico/tecnológicas que estão à disposição do Design(er) de maneira cada vez mais rápidas e inovadoras. Formar Designers sem Laboratórios é como formar Médicos sem Hospitais-Escola, neste sentido faz-se necessário a abertura de portas e consciências que 
permitam a agregação de conhecimentos, pois assim poderemos potencializar a criatividade e a inovação nos projetos e nas pesquisas em design. Sendo assim, unir diferentes campos de fala significa poder dialogar e interagir com diversas áreas de conhecimento. Essa conexão dos diversos saberes e competências não é uma empreitada simples e depende fortemente de pessoal comprometido, com experiência em pesquisa e gestão do conhecimento. Neste sentido, é crucial que os docentes estejam empenhados em não deixar que nossos alunos (de graduação e de pósgraduação) percam a sinergia do "efeito dos colegas". É fundamental que nós professores pesquisadores possamos garantir espaços de vivência laboratorial cada vez mais atraentes com presença de pessoal advindos de diferentes áreas para que tenhamos um ambiente de trabalho diversificado, fundamental na atual conjuntura do ensino e da pesquisa multidimensional em Design.

\section{Agradecimentos}

Os autores agradecem o apoio do Conselho Nacional de Desenvolvimento Científico e Tecnológico (CNPq) processo número 301843/2016-3, bem como o apoio da Coordenação de Aperfeiçoamento de Pessoal de Nível Superior - Brasil (CAPES) - Código de Financiamento 001.

\section{Referências}

BARBIAN, T. Design e tecnologia : gravação a laser de desenhos no arenito. $2015.127 \mathrm{f}$. Dissertação (Mestrado) - Universidade Federal do Rio Grande do Sul, Porto Alegre, 2015.

BLOCH, D. (ED.). Grenoble, cité internationale, cité d'innovations - rêves et réalités. Grenoble (França): Presses Universitaires de Grenoble, 2011.

BRASIL. Portaria $\mathrm{n}^{\circ}$ 389, de 23 de março de 2017. Dispõe sobre o mestrado e doutorado profissional no âmbito da pós-graduação stricto sensu. . 2017, p. seção 1, Brasília, DF, n. 58, páginas 61, 24 março 2017.

BRESSAN, F. Design e tecnologia : estratégias generativas expressivas em superfície e volumetria. 2018. $230 \mathrm{f}$. Tese (Doutorado) - Universidade Federal do Rio Grande do Sul, Porto Alegre, 2018.

BUGIN, L. A. K. et al. Exploration of data-driven midsole algorithm design based in biomechanics data and Voronoi 3D to digital manufacturing. Design e Tecnologia, v. 10, n. 21, p. 01-10, 21 dez. 2020. DOI: https://doi.org/10.23972/det2020iss21pp01-10

CHIEN, Y. H.; CHU, P. Y. The Different Learning Outcomes of High School and College Students on a 3D-Printing STEAM Engineering Design Curriculum. International Journal of Science and Mathematics Education, v. 16, n. 6, p. 1047-1064, 1 ago. 2018. DOI: https://doi.org/10.1007/s10763-017-9832-4

EXPERIMENTA. LE SALON - La Biennale Arts Sciences. Disponível em: <https://www.experimenta.fr/installations/>. Acesso em: 17 fev. 2021.

FAGUNDES, H. A. V. et al. Superfícies bioinspiradas: estudo de caso para aplicações em painéis para fachadas de edifícios. Educação Gráfica (UNESP Bauru), v. 24, n. 3, p. 161179, 2020.

FREITAS, L. DE; MORIN, E.; NICOLESCU, B. Carta da Transdisciplinaridade. Convento da Arrábida (Portugal), 6 nov. 1994. Disponível em: <http://cetrans.com.br/assets/docs/CARTA-DA-TRANSDISCIPLINARIDADE1.pdf〉.

Acesso em: 25 fev. 2021 
GE, X.; IFENTHALER, D.; SPECTOR, J. M. (EDS.). Emerging Technologies for STEAM Education. Cham: Springer International Publishing, 2015.

GOBBI, M. M. Design e tecnologia : resgatando ícones de inovação em móveis para escritórios via arqueologia industrial. 2011. 145 f. Dissertação (Mestrado) - Universidade Federal do Rio Grande do Sul, Porto Alegre, 2011.

GUYOTTE, K. W. et al. Steam as Social Practice: Cultivating Creativity in Transdisciplinary Spaces. Art Education, v. 67, n. 6, p. 12-19, 16 nov. 2014. DOI: https://doi.org/10.1080/00043125.2014.11519293

HALLINEN, J. STEM. Disponível em: <https://www.britannica.com/topic/STEMeducation>. Acesso em: 16 out. 2020.

HARRER, B.; KASTNER, J. X-ray Microtomography: Characterisation of Structures and Defect Analysis. In: Advanced Structured Materials. [s.1.] Springer Verlag, 2011. v. 10p. 119-149. DOI: https://doi.org/10.1007/978-3-642-17782-8_6

HARRIS, A.; DE BRUIN, L. R. Secondary school creativity, teacher practice and STEAM education: An international study. Journal of Educational Change, v. 19, n. 2, p. 153-179, 8 maio 2018. DOI: https://doi.org/10.1007/s10833-017-9311-2

KINDLEIN JÚNIOR, W. O Brilho do Olhar da Pós-Graduação Sparkling Eyes of the Postgraduate. Estudos em Design, v. 22, n. 3, p. 115-129, 28 set. 2014. DOI: https://doi.org/10.35522/EED.V22I3.124

KINDLEIN JÚNIOR, W.; GUANABARA, A. S. Methodology for product design based on the study of bionics. Materials \& Design, v. 26, n. 2, p. 149-155, abr. 2005. DOI: https://doi.org/10.1016/j.matdes.2004.05.009

LEWIS, A. L. Putting the "H" in STEAM: Paradigms for Modern Liberal Arts Education. In: Emerging Technologies for STEAM Education. [s.1.] Springer International Publishing, 2015. p. 259-275. DOI: https://doi.org/10.1007/978-3-319-02573-5_14

LIAO, C. From Interdisciplinary to Transdisciplinary: An Arts-Integrated Approach to STEAM Education. Art Education, v. 69, n. 6, p. 44-49, 18 nov. 2016. DOI: https://doi.org/10.1080/00043125.2016.1224873

MALLON, W.; BUNTON, S. The Functions of Centers and Institutes in Academic Biomedical Research. Analysis in Brief, v. 5, n. 1, p. 1-2, 1 jun. 2005.

MARMON, M. The Emergence of the Creativity in STEM: Fostering an Alternative Approach for Science, Technology, Engineering, and Mathematics Instruction Through the Use of the Arts. In: STEAM Education. Cham: Springer International Publishing, 2019. p. 101-115. DOI: https://doi.org/10.1007/978-3-030-04003-1_6

NOGUEIRA, F. M. et al. Heat transfer in the tank-inflorescence of Nidularium innocentii (Bromeliaceae): Experimental and finite element analysis based on X-ray microtomography. Micron, v. 124, p. 102714, set. 2019. DOI: https://doi.org/10.1016/j.micron.2019.102714

OGNIEWICZ, R.; ILG, M. Voronoi skeletons: theory and applications. Proceedings 1992 IEEE Computer Society Conference on Computer Vision and Pattern Recognition. Anais... IEEE Comput. Soc. Press, 1992

PALOMBINI, F. L. et al. Design, biônica e novos paradigmas: uso de tecnologias 3D para análise e caracterização aplicadas em anatomia vegetal. Design e Tecnologia, v. 7, n. 13, p. 46, 30 jun. 2017. DOI: https://doi.org/10.23972/det2017iss13pp46-56

PALOMBINI, F. L. et al. Design-Aided Science: o designer como promotor de tecnologias 3D para inovação em pesquisa científica. Revista Educação Gráfica, v. 22, n. 3, p. 169-186, 2018a.

PALOMBINI, F. L. et al. Materiais e Biônica: sob a Ótica da Análise de Elementos Finitos 
Baseada em Imagens de Microtomografia de Raios X. In: ARRUDA, A. J. V. (Ed.). Métodos e Processos em Biônica e Biomimética: a Revolução Tecnológica pela Natureza. São Paulo: Editora Blucher, 2018b. p. 245-260. DOI: https://doi.org/10.5151/9788580393491-15

PALOMBINI, F. L. Diretrizes para pesquisas em materiais vegetais com análises por elementos finitos baseadas em microtomografia de raios $\mathrm{X}$ e implicações para projetos de biônica em design e engenharia. 2020. 196 f. Tese (Doutorado) - Universidade Federal do Rio Grande do Sul, Porto Alegre, 2020.

PALOMBINI, F. L. et al. Combining numerical models and discretizing methods in the analysis of bamboo parenchyma using finite element analysis based on X-ray microtomography. Wood Science and Technology, v. 54, n. 1, p. 161-186, 14 jan. 2020a. DOI: https://doi.org/10.1007/s00226-019-01146-4

PALOMBINI, F. L. et al. Biomimetic systems and design in the 3D characterization of the complex vascular system of bamboo node based on X-ray microtomography and finite element analysis. Journal of Materials Research, v. 35, n. 8, p. 842-854, 28 abr. 2020b. DOI: https://doi.org/10.1557/jmr.2019.117

PERIGNAT, E.; KATZ-BUONINCONTRO, J. STEAM in practice and research: An integrative literature review. Thinking Skills and Creativity, v. 31, p. 31-43, 1 mar. 2019. DOI: https://doi.org/10.1016/j.tsc.2018.10.002

PNPG - PLANO NACIONAL DE PÓS-GRADUAÇÃO (2011-2020). Relatório Final de acompanhamento da Comissão Especial de Acompanhamento. Brasília: fevereiro, 2017.

QUIGLEY, C. F.; HERRO, D.; BAKER, A. Moving Toward Transdisciplinary Instruction: A Longitudinal Examination of STEAM Teaching Practices. In: STEAM Education. Cham: Springer International Publishing, 2019. p. 143-164. DOI: https://doi.org/10.1007/978-3-03004003-1_8

SCHUMMER, J. Interdisciplinary Issues in Nanoscale Research. In: BAIRD, D.; NORDMANN, A.; SCHUMMER, J. (Eds.). Discovering the Nanoscale. Amsterdam: IOS Press, 2004. p. 9-20.

SHATUNOVA, O. et al. STEAM as an Innovative Educational Technology. Journal of Social Studies Education Research, v. 10, n. 2, p. 131-144, 2019.

STEAM EDUCATION. STEAM. Disponível em: 〈https://steamedu.com/>. Acesso em: 17 fev. 2021.

STEIN, E. History of the Finite Element Method - Mathematics Meets Mechanics - Part I: Engineering Developments. In: Lecture Notes in Applied Mathematics and Mechanics. [s.1.] Springer, Berlin, Heidelberg, 2014. v. 1p. 399-442. DOI: https://doi.org/10.1007/978-3642-39905-3_22

STOCK, S. R. MicroComputed tomography : methodology and applications. Boca Raton, Florida, USA: CRC Press, 2009.

WALLISS, J.; RAHMANN, H. Landscape Architecture and Digital Technologies. Abingdon, Oxon [UK]; New York [USA]: Routledge, 2016.

ZIENKIEWICZ, O. C.; TAYLOR, R. L.; ZHU, J. Z. The finite element method : its basis and fundamentals. 7. ed. Oxford: Butterworth-Heinemann, 2013. 


\section{Sobre os autores}

\section{Wilson Kindlein Junior}

Professor titular do Departamento de Engenharia de Materiais da UFRGS. É bolsista de Produtividade em Pesquisa PQ-1A (Design Industrial) do CNPq. Atua no Laboratório de Design e Seleção de Materiais / UFRGS. Engenheiro Mecânico pela UFRGS (1988), realizou estágios em Design Industrial na França e possui titulação de Mestrado (1993) e Doutorado (1999) em Engenharia pela UFRGS. É Prof. orientador do PGDesign / UFRGS e do PPGE3M / UFRGS. https://orcid.org/0000-0002-5939-4126

\section{Felix Bressan}

Professor adjunto do Departamento de Artes Visuais da UFRGS - Escultura. Prof. colaborador do Laboratório de Design e Seleção de Materiais / UFRGS. Bacharel em Artes Plásticas, Escultura UFRGS (1992). Mestre em Artes Visuais, ênfase em Poéticas Visuais (1996), pelo PPGAV / UFRGS. Doutor (2018) em Design pelo Programa de Pós-Graduação em Design (PGDesign / UFRGS). Produção escultórica integra importantes coleções privadas e públicas. https://orcid.org/0000-0003-1040-5477

\section{Felipe Luis Palombini}

Designer de produto (2015), Mestre (2016) e Doutor (2020) em Design pelo Programa de PósGraduação em Design (PGDesign / UFRGS). Coordenou e ministrou cursos de extensão sobre Tecnologias 3D para a pós-graduação na UFRGS e foi professor substituto no Departamento de Desenho Industrial da UFSM. Trabalha nas áreas de biônica e caracterização de materiais, com ênfase em Análise por Elementos Finitos baseada em imagens de Microtomografia de Raios X. https://orcid.org/0000-0002-2112-6695 